\title{
Prototypes of attractors in four dimensions
}

\author{
Baier, G.; Thomsen, Jesper Skovhus
}

Published in:

Physical Review E. Statistical, Nonlinear, and Soft Matter Physics

Link to article, DOI:

10.1103/PhysRevE.48.R4172

Publication date:

1993

Document Version

Publisher's PDF, also known as Version of record

Link back to DTU Orbit

Citation (APA):

Baier, G., \& Thomsen, J. S. (1993). Prototypes of attractors in four dimensions. Physical Review E. Statistical, Nonlinear, and Soft Matter Physics, 48(6), R4172-R4174. https://doi.org/10.1103/PhysRevE.48.R4172

\section{General rights}

Copyright and moral rights for the publications made accessible in the public portal are retained by the authors and/or other copyright owners and it is a condition of accessing publications that users recognise and abide by the legal requirements associated with these rights.

- Users may download and print one copy of any publication from the public portal for the purpose of private study or research.

- You may not further distribute the material or use it for any profit-making activity or commercial gain

- You may freely distribute the URL identifying the publication in the public portal

If you believe that this document breaches copyright please contact us providing details, and we will remove access to the work immediately and investigate your claim 


\title{
Prototypes of attractors in four dimensions
}

\author{
G. Baier \\ Institute for Chemical Plant Physiology, University of Tübingen, D-72076 Tübingen, Germany \\ J. S. Thomsen \\ Chaos Group, Physics Department, Technical University of Denmark, DK-280o Lyngby, Denmark
}

(Received 16 September 1993)

\begin{abstract}
We study an extension of Duffing's equation to three variables with external forcing. Starting from a phase-space preserving chaos, three prototypes of chaotic attractors with a dimension larger than 3 can be derived. We provide examples of hyperchaos and a "bifractal" in a four-dimensional flow. The second-order Poincaré cross section of hyperchaotic flow is qualitatively equivalent to the first-order cross section of Ueda's attractor with the same forcing.
\end{abstract}

PACS number(s): 05.45.+b, 02.60.-x

\section{INTRODUCTION}

A four-dimensional flow can be realized either as a system of four autonomous ordinary differential equations or as a three-variable system with external forcing. The asymptotic dynamics of such systems can be described by the four Lyapunov characteristic exponents (LCE's). Leaving aside the zero exponent in the direction of the trajectory (or forcing, respectively), the three remaining LCE's can be used to classify the possible types of behavior. Two criteria have been introduced for the distinction of chaotic behavior. (i) Exponents can be either positive or nonpositive. Using the spectrum of ordered LCE's, $\lambda_{1} \geq \lambda_{2} \geq \lambda_{3}, \sum_{i=1}^{3} \lambda_{i}<0$, there are thus two possibilities in the case of four-dimensional flows: $\lambda_{1}>0, \lambda_{2} \leq 0$ (chaos), and $\lambda_{1}, \lambda_{2}>0$ (hyperchaos). Two examples of autonomous four-variable systems exhibiting hyperchaos have been described $[1,2]$. However, in these examples outside the range of parameter with hyperchaos the trajectory either escapes to a stable steady state or to infinity. There seem to be no detailed studies of prototypic four-dimensional flows which illuminate generic features; e.g., the bifurcation sequences to hyperchaos. Therefore findings of two positive LCE's in experimental time series have had no deeper explanation in terms of model equations so far [3-5]. (ii) Two exponents of opposite sign compete with each other. This allows for three cases of chaos with one positive Lyapunov exponent: (A) $\left(\lambda_{1}+\lambda_{2}\right)<0$, ordinary chaos with a Lyapunov dimension $2<D_{L}<3$; (B) $\left(\lambda_{1}+\lambda_{2}\right)>0$ and $\left(\lambda_{1}+\lambda_{3}\right)<0$, chaos with a Lyapunov dimension $3<D_{L}<4$ and a sheetlike cross section [6]; (C) $\left(\lambda_{1}+\lambda_{2}\right)>0$ and $\left(\lambda_{1}+\lambda_{3}\right)>0$, chaos with a Lyapunov dimension $3<D_{L}<4$ and a second direction of fractalization in the cross section (a "bifractal" [7]). These types have been characterized in a three-dimensional nonlinear map [8]. We are not aware of simple generic four-dimensional (4D) flows where all these types have been found.

\section{RESULTS}

We provide a prototypic ordinary differential equation where all four types of chaotic attractors including hy- perchaos can be observed due to the systematic design of the system:

$$
\begin{aligned}
& \dot{x}=y \\
& \dot{y}=k_{1} y-x^{3}+B \cos (t)-C_{1} z, \\
& \dot{z}=k_{2} z-C_{2} x
\end{aligned}
$$

with $x, y, z, k_{1}, k_{2}, B, C_{1}, C_{2} \in \mathbb{R}$.

For $C_{1}=0$ the subsystem $x, y$ is Duffing's equation as studied by Ueda [9]. Variable $z$ possesses one term which contributes to the trace of the Jacobian governed by parameter $k_{2}$, and $C_{1}$ and $C_{2}$ are used to linearly couple Duffing's oscillator to variable $z$. When $k_{1}=k_{2}=$ $C_{1}=0$, variable $z$ is coupled passively to the subsystem $x, y$ and Duffing's oscillator is known to display volumepreserving chaotic orbits [10]. The spectrum of LCE's of this volume-preserving chaos is $(+, 0,-)$. Here, the zero exponent reflects the passively coupled third variable.

Parameters $k_{1}$ and $k_{2}$ (together with nonzero coupling $C_{1}$ ) can now be exploited to tune this spectrum in three different directions to yield attracting solutions. The trace of the Jacobian is globally constant and equals $k_{1}+k_{2}$; therefore $k_{1}+k_{2}<0$ is required for attractors.

$$
\text { A. } k_{1}<0 \text { and } k_{2}>0
$$

For numerically small values of $k_{1}$ and $k_{2}$ the LCE spectrum reflects the deviation from the conservative case. Negative $k_{1}$ (i.e., the negative contribution to the trace of the Jacobian) will increase the absolute value of $\lambda_{3}$; the negative exponent of the former conservative case moves farther from zero. Positive $k_{2}$ brings a positive contribution to the trace of the Jacobian. Due to this expansion in variable $z$ (governed by parameter $k_{2}$ ) a second positive Lyapunov exponent can arise from the former zero exponent and therefore hyperchaos can be found. For $k_{1}=-0.04, k_{2}=0.02, B=34, C_{1}=-0.11$, and $C_{2}=3.0$, we have found hyperchaos to exist. The LCE's are $(0.1634,0.0237,-0.2180)$ yielding a Lyapunov dimension of 3.858. Figure 1 shows a second-order cross section of this hyperchaotic flow. The plot is qualitatively equivalent to the first-order cross section of Ueda's 


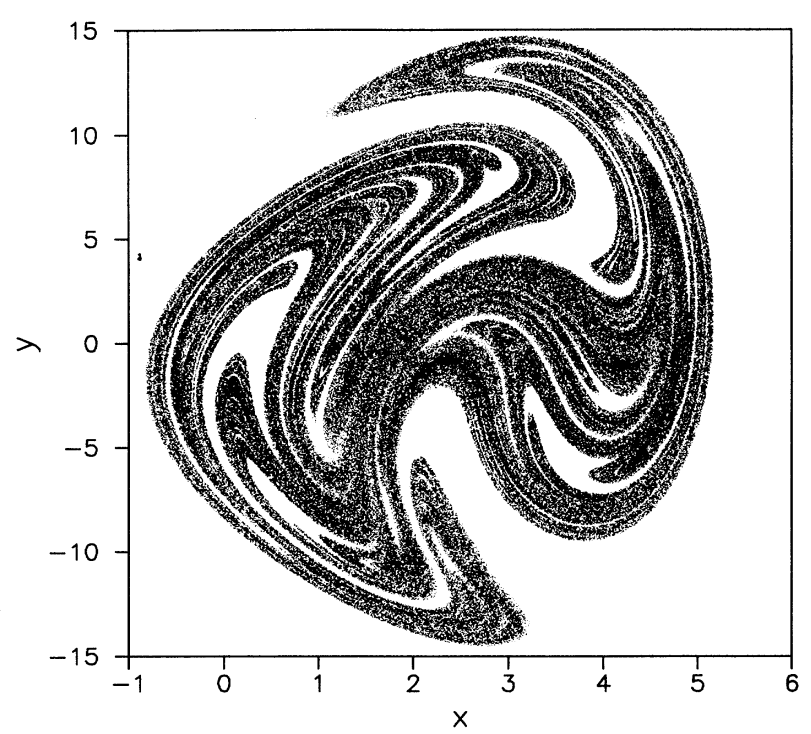

FIG. 1. Second-order cross section of hyperchaotic flow for parameters as given in the text. The first-order cross section was taken at intervals of $2 \pi$. For the second cut an $\epsilon$ band of \pm 1 around $z=0$ was applied. The $z$ variable lies in the range $[-150 ; 150] .261301$ points are drawn.

attractor at the same forcing amplitude and frequency (see [10]). This confirms that the building-block principle for higher-dimensional flows [11] works. Hyperchaotic solutions are found in a finite parameter range.

We have performed a $1 \mathrm{D}$ continuation on Eq. (1) with different fixed values of $k_{1}$ and varying values of $B$ (other parameters as given above for hyperchaos). In the $\left(B, k_{1}\right)$ plane around the region with hyperchaos we found a "bump" of torus bifurcations similar to the pitchfork "bump" from Ueda's system (denoted "IV" in [10]). Also there is evidence of a cusplike saddle-node bifurcation curve, but in our model this curve is interrupted by pitchfork bifurcations. The pitchfork bifurcations keep existing for quite high values of the damping $k_{1}$. No evidence of period-doubling bifurcations was found.

\section{B. $k_{1}<0$ and $k_{2}<0$}

With both $k_{1}$ and $k_{2}$ negative both $\lambda_{2}$ and $\lambda_{3}$ become more negative compared to the conservative case. For numerically small values of $k_{1}$ and $k_{2}$ the chaotic solutions can be characterized by $\left(\lambda_{1}+\lambda_{2}\right)>0$ and $\left(\lambda_{1}+\lambda_{3}\right)<0$, and the resulting attractors thus have a Lyapunov dimension $3<D_{L}<4$ and a smooth sheetlike cross section. This type of chaotic behavior is described in [6].

Increasing dissipation, e.g., by decreasing parameter $k_{2}$, simple chaos with $\left(\lambda_{1}+\lambda_{2}\right)<0$ and with a Lyapunov dimension $2<D_{L}<3$ can be found. Here the cross section is a Cantor set of folded lines.

$$
\text { C. } k_{1}>0 \text { and } k_{2}<0
$$

For small values of both parameters the negative Lyapunov exponent of the conservative case will decrease in absolute value (move closer to zero) due to positive $k_{1}$, but the zero exponent of the conservative case gets negative due to negative $k_{2}$. With $k_{1}+k_{2}<0$ an attractor will result which fulfills both $\left(\lambda_{1}+\lambda_{2}\right)>0$ and $\left(\lambda_{1}+\lambda_{3}\right)>0$. The stroboscopic plot of this attractor resembles the hyperchaotic case, but the different spectral composition leads to a new type of fractal structure. Exemplary parameter values are $k_{1}=0.02, k_{2}=-0.04$, $B=34, C_{1}=5.9$, and $C_{2}=0.1$. This attractor has a Lyapunov spectrum of $(0.1940,-0.0479,-0.1752)$, and thus a dimension of $D_{L}=3.834$.

\section{DISCUSSION}

The dynamic behavior of Duffing's oscillator was known as a function of the dissipation parameter from the study in [10]. The key to the results presented above was the set of parameters that gives rise to a positive LCE in Duffing's equations. A linearly coupled third variable creates a new direction with zero divergence in the phase-space preserving case $\left(k_{1}+k_{2}=0\right)$. Then the introduction of nonzero $k_{1}$ and $k_{2}$ with $k_{1}+k_{2}<0$ allows the manipulation of the two nonpositive exponents. In particular, $k_{2}>0$ creates a new direction of expansion orthogonal to the chaotic divergence of the conservative system. The single nonlinearity with the correct symmetry (third order) is capable of keeping this (second) expansion bounded, and stable hyperchaotic solutions are obtained for small positive values of $k_{2}$. Note that Duffing's equation is a physical model and was not tailored to create hyperchaos as the Rössler system. Hyperchaos in the context of Duffing's oscillator was also observed by Kapitaniak [12], but periodic forcing of four coupled nonlinear oscillators was required to achieve this result.

If $k_{2}<0$, a new direction of convergent behavior is created (compared to the volume-preserving case) and a stable attractor results immediately. For small negative values of $k_{2}$, the exponent resulting from this convergent behavior is (in absolute value) smaller than the positive exponent from the mean divergence. This leads to the familiar situation where on the average one local rate of convergence to the attractor is weaker (in absolute value) than the local rate of divergence [8]. The result is an attractor of Lyapunov dimension larger than 3 .

Parameter $k_{1}$ can be used to adjust the value of the more negative Lyapunov exponent. If $k_{1}>0$, then the third Lyapunov exponent $\lambda_{3}$ can be managed to move closer to zero and for small positive values of $k_{1}$ condition $\left(\lambda_{1}+\lambda_{3}\right)>0$ is satisfied for a finite range of parameter. This means there is a second mean value of convergence in the system which is smaller (in absolute value) than the mean divergence of the chaos. Thus there is a second situation where the local rate of convergence is on the average smaller (in absolute value) than the local rate of divergence at that point. In contrast to the first case there can be no second increase of the attractor dimension over the next integer because phase space is only four dimensional. Now the competition of rate of convergence with the rate of divergence leads to a second direc- 
tion of fractalization orthogonal to the first fractalization caused by the stretching-and-folding operation in phase space. This feature has been observed only numerically so far and an analytic proof seems out of reach. However, the same transition has been shown for a nonlinear threedimensional map in a situation close to invertibility [8] and the transition criteria from smooth to bifractal attractors could be given analytically in a piecewise linear map with explicitly controllable rates of convergence and divergence [13].

To summarize we conjecture that, according to our classification, there are three types of attractors with
Lyapunov dimension $3<D_{L}<4$, which are prototypic for four-variable flows.

\section{ACKNOWLEDGMENTS}

Simulations were performed using SimPack software by Knudsen, Feldberg, and Thomsen, performing a 5th to 6th order Runge-Kutta integration method with stepsize control. We thank Erik Mosekilde, Otto Rössler, and Carsten Knudsen for stimulation. G.B. is supported by the Deutsche Forschungsgemeinschaft.
[1] O.E. Rössler, Phys. Lett. A 71, 155 (1979).

[2] H. Killory, O.E. Rössler, and J.L. Hudson, Phys. Lett. A 122, 341 (1987).

[3] R. Stoop and P.F. Meier, J. Opt. Soc. Am. B 5, 1037 (1988).

[4] R. Stoop, J. Peinke, J. Parisi, B. Röhricht, and R.P. Huebener, Physica D 35, 425 (1989).

[5] M. Eiswirth, Th.-M. Kruel, G. Ertl, and F.W. Schneider, Chem. Phys. Lett. 193, 305 (1992).

[6] J. Kaplan and J.A. Yorke, in Functional Differential Equations and Fixed Points, edited by H.-O. Peitgen and H.-O. Walther, Lecture Notes in Mathematics Vol. 730 (Springer, Berlin, 1979).

[7] B. Röhricht, W. Metzler, J. Parisi, J. Peinke, W. Beau, and O.E. Rössler, in The Physics of Structure Formation, edited by W. Güttinger and G. Dangelmayr (Springer, New York, 1987).

[8] G. Baier and M. Klein, Phys. Lett. A 177, 32 (1993).

[9] Y. Ueda, in New Approaches to Nonlinear Problems, edited by P.J. Holmes (SIAM, Philadelphia, 1980).

[10] Y. Ueda, J.S. Thomsen, J. Rasmussen, and E. Mosekilde, in Mathematical Research 72, edited by $\mathrm{H}$. Kreuzer and G. Schmidt (Akademie Verlag, Berlin, 1993).

[11] M. Klein and G. Baier, in A Chaotic Hierarchy, edited by G. Baier and M. Klein (World Scientific, Singapore, 1991).

[12] T. Kapitaniak, Phys. Rev. E 47, 2976 (1993).

[13] G. Baier, A. Kittel, and M. Klein, Phys. Rev. E 48, 3409 (1993). 